\title{
DNA Fingerprinting of Closely Related Cultivars of Sweet Cherry
}

\author{
Paul A. Wiersma ${ }^{1}$ \\ Summerland Research and Development Centre, Agriculture and Agri-Food Canada, 4200 Highway \\ 97, Summerland, BC VOH 1Z0, Canada \\ Deniz Erogul \\ Department of Horticulture, Faculty of Agriculture, University of Ege, Bornova, Izmir 35100, Turkey
}

\author{
Shawkat Ali \\ Kentville Research and Development Centre, Agriculture and Agri-Food Canada, 32 Main Street, \\ Kentville, NS B4N 1J5, Canada
}

\begin{abstract}
Additional index words. Prunus avium, simple sequence repeats, single nucleotide polymorphism, heterozygosity, discrimination power

AbStract. Simple sequence repeat (SSR) and single nucleotide polymorphism (SNP) markers were evaluated in an effort to reliably DNA fingerprint sweet cherry (Prunus avium L.) cultivars and advanced selections from the breeding program at the Summerland Research and Development Center (Summerland, BC, Canada). SSR markers were found that differentiated the 35 cultivars and selections tested. However, groups of cultivars closely related to the parental cultivars, Lapins and Sweetheart, were differentiated by only a few SSR markers each. These last few markers were discovered by specifically screening within these small groups of cultivars and the resulting markers had lower discriminating power $\left(D_{j}\right)$ statistics within the full set of 35 cultivars and selections. To further characterize the differences in one of these closely related groups, SNP markers were identified in the cultivar Sweetheart and an analysis was made of how these markers segregated into three of its open-pollinated progeny. Large blocks of the 'Sweetheart' genome (34\%) did not contain informative SNP markers, which was consistent with its ancestry where the cultivar Van is both a parent and grandparent. The three progeny cultivars differed from 'Sweetheart' at $14 \%$, $31 \%$, and $29 \%$ of the 3011 SNP positions tested. These were located in blocks of linked haplotypes covering from 2.5 to 20 million bps each and were distinct for the three cultivars. The cultivar Staccato ${ }^{\circledR}$, which required the most effort for SSR marker discrimination, also had the lowest number of SNP position differences from 'Sweetheart' (14\%). These informative SNP markers were located in only five small regions of the sweet cherry genome, which also contained the discriminating SSR markers and provides an explanation for the difficulty of locating SSR markers for this cultivar. In addition to clearly differentiating these cultivars, this SNP analysis shows the level of variation expected within this closely related group.
\end{abstract}

An international breeding program has an ongoing requirement to reliably identify their cultivars to maintain correct material during propagation and for enforcement of plant breeders' rights. The sweet cherry breeding program from the Summerland Research and Development Center of AgricultureAgri-Food Canada (SuRDC, Summerland, BC, Canada) and their official licensing organization, Summerland Varieties Corporation, continue to identify new cultivars, which require the precision of identification that is available with DNA marker technologies. Although a similar study had been carried out with amplified fragment length polymorphism (AFLP) markers (Zhou et al., 2002) it was determined that the level of hands-on expertise required to run and evaluate these markers was too high for occasional use, also noted by Noli et al. (2013). In the three decades since DNA markers were first introduced, there has been rapid development of marker

Received for publication 11 May 2018. Accepted for publication 27 June 2018. Turkish Council of Higher Education supported the stay of Deniz Erogul in Canada. We thank Guus Bakkeren and Amritpal Singh, Agriculture and AgriFood Canada, Summerland Research and Development Centre for critical review of the manuscript and the technical assistance of Virginia Dickison, Nadia Sokal, and student Alix Wong and members of the sweet cherry breeding program.

${ }^{1}$ Corresponding author. E-mail: paul.wiersma@agr.gc.ca. technologies, particularly with the advent of DNA amplification in polymerase chain reaction (PCR). When this project was started, SSR markers were the marker of choice (Nybom et al., 2014), with extensive sets of markers developed for sweet cherry and other closely related Prunus L. species (Jung et al., 2014; Olmstead et al., 2008). Previous studies with sweet cherry showed that a few, highly informative, markers were sufficient to differentiate a wide range of cultivars (Dirlewanger et al., 2002; Fernandez i Marti et al., 2012; Pedersen, 2006; Wunsch and Hormaza, 2004). This method was, therefore, tested on 35 cultivars (i.e., named cultivars and advanced selections) from the SuRDC program, some of which were difficult to differentiate by AFLP (Zhou et al., 2002). Many of these cultivars are currently licensed, or are advanced selections with the potential for licensing, and are expected to be propagated and planted worldwide, making a reproducible means of identification at the DNA level an essential tool.

\section{Materials and Methods}

Sample PREParation. Reference trees for the 35 sweet cherry cultivars and selections in Table 2 were identified using the records of the sweet cherry breeding program and located 
within the orchards of the SuRDC. Young expanding leaf tissue was collected from mature sweet cherry trees in spring and samples were frozen and stored at $-80^{\circ} \mathrm{C}$ until extraction. DNA extraction was by the method of Zhou et al. (2002) and RNA extraction was by a modification of the method of Fils-Lycaon et al. (1996). For RNA extraction, $0.5 \mathrm{~g}$ of frozen powdered tissue was quickly mixed with $1.2 \mathrm{~mL}$ of extraction buffer (0.2 $\mathrm{M}$ glycine, $0.1 \mathrm{M}$ disodium phosphate, $0.6 \mathrm{M}$ sodium chloride, $\mathrm{pH} 9.5) ; 66 \mu \mathrm{L}$ of 2 -mercaptoethanol; $66 \mu \mathrm{L}$ of $20 \%$ (w/v) sodium dodecylsulphate; and $1 \mathrm{~mL}$ of phenol (equilibrated greater than $\mathrm{pH} 7.6$ with Tris-HCl). After centrifugation, the clear aqueous layer was removed to a clean tube and ethanol added to a final concentration of $30 \%(\mathrm{v} / \mathrm{v})$. This RNA solution was pipetted into a disposable plastic column (Poly-Prep; Bio-Rad, Hercules, CA) containing a bed of $\approx 200 \mathrm{mg}$ of CC41 cellulose (Whatman, Maidstone, England) suspended in 1X STE (100 mm NaCl, $50 \mathrm{~mm}$ Tris- $\mathrm{HCl} \mathrm{pH} 8.0,1 \mathrm{~mm}$ EDTA) and 30\% ethanol. The column was attached to a vacuum manifold and washed with $40 \mathrm{~mL}$ of $1 \mathrm{X}$ STE-30\% ethanol to remove DNA and contaminants. The RNA was eluted from the column by centrifugation with three washes of RNase-free water ( $550 \mu \mathrm{L}$ total); cellulose fines were removed by centrifugation and the RNA recovered by ethanol precipitation.

SSR ANALYSIS. SSR primer sequences were as referenced from Olmstead et al. (2008) and the Genome Database for Rosaceae (GDR; Jung et al., 2014). PCR reactions, in a total volume of $25 \mu \mathrm{L}$, contained $2.5 \mathrm{mM} \mathrm{MgCl}_{2}, 0.3 \mu \mathrm{M}$ each of forward and reverse primer, $200 \mu \mathrm{M}$ dNTPs, $0.5 \mathrm{U}$ of AmpliTaq ${ }^{\circledR}$ Gold DNA polymerase (Applied Biosystems, Foster City, CA), and 1X PCR Buffer II (supplied with the enzyme). Dilutions of genomic DNA were optimized for each primer pair with input DNA ranging from 1 to $50 \mathrm{ng}$ per reaction. Thermocycling conditions were $95^{\circ} \mathrm{C}$ for $10 \mathrm{~min}$; 35 cycles of $94^{\circ} \mathrm{C}$ for $1 \mathrm{~min}, 55^{\circ} \mathrm{C}$ for $1 \mathrm{~min}$, and $72^{\circ} \mathrm{C}$ for $1 \mathrm{~min}$; and a final extension at $72{ }^{\circ} \mathrm{C}$ for $5 \mathrm{~min}$. SSR reactions were electrophoresed on polyacrylamide gels and silver stained following the detailed protocol of George and Regalado (2003). SSR statistics were evaluated in a computer spreadsheet (Excel; Microsoft, Redmond, WA) from the following equations, with $p_{i}$ (or $p_{j}$ ) as the frequency of allele $i$ (or $\left.j\right), g_{i}$ as the frequency of genotype (or pattern) $i$, and $N$ as total number of compared cultivars: gene diversity, $H_{\mathrm{e}}=\sum_{i} p_{i}^{2}$, Nei (1973); direct count (observed) heterozygosity, $H_{\mathrm{o}}=$ number of samples with heterozygous genotypes/total number of samples; probability of identity, $P_{\mathrm{ID}}=\sum_{i} p_{i}^{4}+\sum_{i} \sum_{j}\left(2 p_{i} p_{j}\right)^{2}$, with $i<j$ in the second term, Waits et al. (2001); confusion probability $\left(C_{j}\right)$, $C_{j}=\sum_{i} g_{i}^{2}\left(N g_{i}-1\right) /(N-1)$, Tessier et al. (1999); discriminating power, $D_{j}=1-C_{j}$ or $D_{\mathrm{L}}=\mathrm{DP}=1-\sum_{i} g_{i}^{2}$, Tessier et al. (1999), Kloosterman et al. (1993); number of nondifferentiated pairs, $x_{j}=C_{j} \times N(N-1) / 2$ (Tessier et al., 1999). The genotype for each cultivar was compared against the other 34 cultivars in this study using macros in Excel. The spreadsheet calculated the differences between each pair of cultivars as either the number of bands or the number of genotypes. Total number of comparisons for the 35 cultivars was 595 (35 choose 2) with each comparison scored as either "same," with all bands or genotypes identical between the two cultivars or "diff," with at least one different band or genotype.
Single NUCleotide POLYMorphism anAlysis. RNA-Seq libraries were prepared from young leaf mRNA of the cultivars Sweetheart and three of its offspring Staccato ${ }^{\circledR}$, Sovereign ${ }^{\mathrm{TM}}$, and Sentennial ${ }^{\mathrm{TM}}$ with a TruSeq kit (Illumina, San Diego, CA) according to the manufacturer's instructions. Two lanes of HiSeq Illumina sequence data were obtained from the BC Cancer Genome Center, Vancouver, BC, producing 200 million $\times 2 \times 75$ bp reads from Sweetheart and 65 million reads for each of the other three cultivars. SNP markers were identified de novo in the 'Sweetheart' RNA-Seq reads using KisSplice (Sacomoto et al., 2012) with options -s $1-\mathrm{k} 51$. The sequence surrounding each SNP (a 103-bp KisSplice fragment) was used to map that sequence within the published sweet cherry genome (Shirasawa et al., 2017) using BLAST+ (Camacho et al. (2009). The RNA-Seq reads for the four cultivars were individually aligned to the sweet cherry genome using BWA MEM (Li and Durbin, 2010). The SNP markers identified in 'Sweetheart' were then evaluated for all four cultivars by running their alignments together through samtools mpileup using the -1 option to specify SNP locations and bcftools view to determine genotypes ( $\mathrm{Li}, 2011)$. This SNP set was refined by requiring a) a good match in the sweet cherry transcriptome; b) that the fragment mapped to a unique location in the eight sweet cherry pseudomolecules (i.e., chromosomes); and c) a raw read depth (vcftools "DP") above 100. A map of the SNP locations for these cultivars on the sweet cherry pseudomolecules was displayed using a modification of the Bio.Graphics.BasicChromosome module from BioPython Pritchard et al. (2006). The SNP sequences, map locations, and genotypes at these positions for 'Sweetheart', 'Staccato ${ }^{\circledR}$, 'Sovereign ${ }^{\mathrm{TM}}$ ', and 'Sentennial ${ }^{\mathrm{TM}}$ ' have been deposited in the GDR marker database.

\section{Results and Discussion}

Making use of the large number of SSR markers previously demonstrated to function in sweet cherry (Jung et al., 2014; Olmstead et al., 2008) we began screening these markers for their ability to differentiate 35 selected cultivars from the SuRDC breeding program. Screening was carried out with small sets of cultivars to evaluate amplification and the resulting fragment sizes and patterns. Selected markers were then assessed on all 35 cultivars, and the three most informative markers (pms3, pms30, and UDP98-022 had the highest number of genotypes, highest DP, lowest $P_{\mathrm{ID}}$, and highest number of differences between cultivars) were sufficient to differentiate 26 of these cultivars (Tables 1 and 2). This is consistent with the results of previous analyses with sweet cherry, where a few highly informative markers were sufficient for discrimination of a broad range of cultivars. However, three groups of closely related cultivars showed no differences with these markers. These groups were: 'Lapins' and two of its progeny, 'Sweetheart' and two of its progeny, and three 'Stella' progeny. Because the difficulty of differentiating the cultivar Sweetheart from its progeny had already been demonstrated (Zhou et al., 2002), additional markers were screened using only eight cultivars, specifically to find differences within these groups. With the addition of three of these markers (BPPCT 014, EMPaS02, and pms67) to the three most informative markers, we were able to differentiate all cultivars except Sweetheart and Staccato ${ }^{\circledR}$. This included 'Sovereign ${ }^{\mathrm{TM}}$ ', 'Sentennial'TM', and 'SPC243', which are also open-pollinated 'Sweetheart' progeny and were expected to pose a similar 


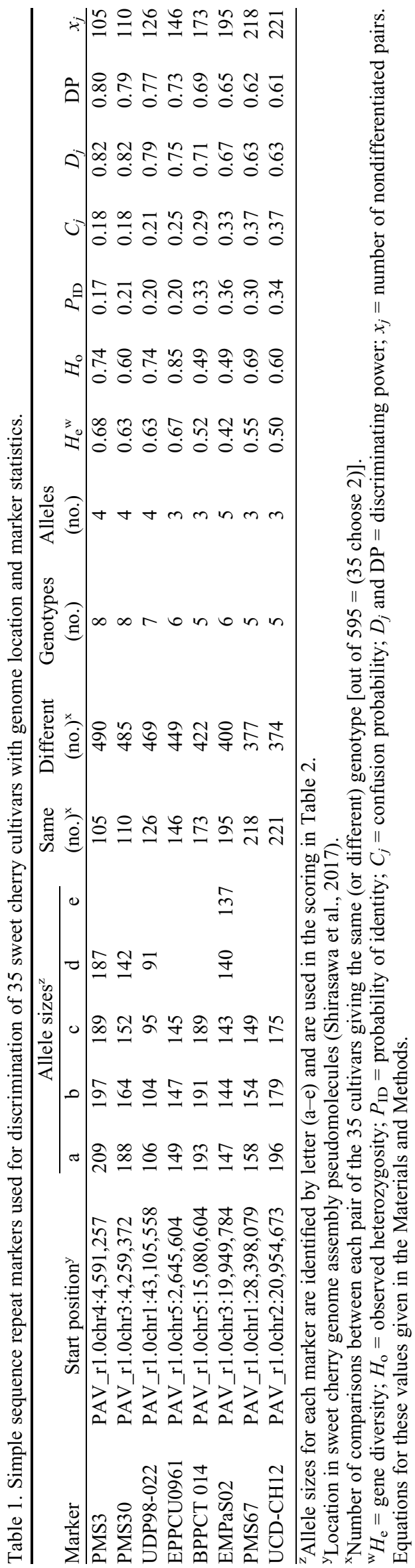

challenge for discrimination. Finally, a broader range of SSR markers was screened using only 'Sweetheart' and 'Staccato ${ }^{\circledR}$ '. The markers EPPCU0961 and UCD-CH12 were identified as differentiating these two cultivars. This final set of eight markers still left three cultivars differing by only one allele and five cultivars with only two differences. In total 108 Prunus SSR markers were screened with 'Sweetheart' and 'Staccato ${ }^{\circledR}$ '. Of these, 27 either did not amplify well (under our conditions) or had large fragment sizes or complex patterns that made scoring difficult (Supplemental Table 1). Of the remaining 81 SSR markers, 41 were homozygous and 40 were heterozygous for Sweetheart. All homozygous markers were identical between 'Sweetheart' and 'Staccato ${ }^{\circledR}$ ' strengthening the suggestion that 'Staccato ${ }^{\circledR}$ ' arose from 'Sweetheart' by a selfpollination event. A different pollen parent would have contributed some alternate alleles in these 41 locations spread across the genome. Only three SSR markers, which were heterozygous in 'Sweetheart', were eventually found to be homozygous in 'Staccato ${ }^{\circledR}$ ' allowing differentiation. This number of differences [7\% (three of 41)] is much lower than expected for a random sexual hybridization event even if it is a self-pollination where a 1:2:1 segregation ratio would be expected (ignoring linkage).

Markers with previously documented discrimination power (DP) for sweet cherry [pms3, pms30, and pms67 from Pedersen (2006); and BPPCT 014 from Dirlewanger et al. (2002)] gave values similar to those found for our set of 35 cultivars $(0.819$ vs. $0.8,0.792$ vs. $0.79,0.5$ vs. $0.62,0.6$ vs. 0.65 , respectively). The values for DP from Pedersen (2006) were from a very small set of sweet cherry cultivars which included Sweetheart and Newstar, which were also in our set of cultivars, whereas the cultivars given in Dirlewanger et al. (2002) were more diverse and would be expected to give a more representative DP value for sweet cherry in general.

Unlike many previous studies with sweet cherry genotyping, where determining the relatedness of the cultivars was a main focus, the ancestry of most of the cultivars in this work are known. This allows us to ascertain the accuracy of the genotyping. Of the 35 cultivars, 10 did not have parental genotypes available so no assessment could be made. Consistent genotypes were found for nine cultivars for which both parents were genotyped (Lapins, Newstar, Santina, Sentennial ${ }^{\mathrm{TM}}$, Sovereign $^{\text {TM }}$, SPC118, SPC232, SPC243, and Staccato ${ }^{\circledR}$ ), assuming self-pollination of self-fertile cultivars. For those cultivars with one of its parents genotyped, there were nine with consistent genotypes (Christalina ${ }^{\mathrm{TM}}$, Satin ${ }^{\mathrm{TM}}$, Sonata ${ }^{\mathrm{TM}}$, SPC133, SPC175, SPC189, SPC221, SPC381, Starblush ${ }^{\top \mathrm{M}}$, Suite Note ${ }^{\mathrm{TM}}$, and Summit). This left five cultivars with genotypes that were not consistent with the recorded parentage. 'Starletta ${ }^{\mathrm{TM}}$ ' had five alleles and 'Samba' had three alleles that are not present in 'Stella', even though these cultivars are recorded as having only 'Stella' in their parentage ['Stella' and '2S-84-10' (selfed 'Stella')]. 'Sumesi' also has a selfed Stella cultivar as a parent ('2S-22-05'), but in this case, the alleles were consistent with 'Stella' but two of them could not have come from the other recorded parent, 'Van'. Similarly, two cultivars in the important 'Van' $\times$ 'Stella' lineage have genotypes which were not consistent; with 'New Moon' having two loci with homozygous alleles that were not present in 'Stella', and 'Sweetheart' having three alleles that could not have come from 'Newstar'. These types of differences could be the result of stray pollen landing on emasculated flowers or labeling errors in the field notes 


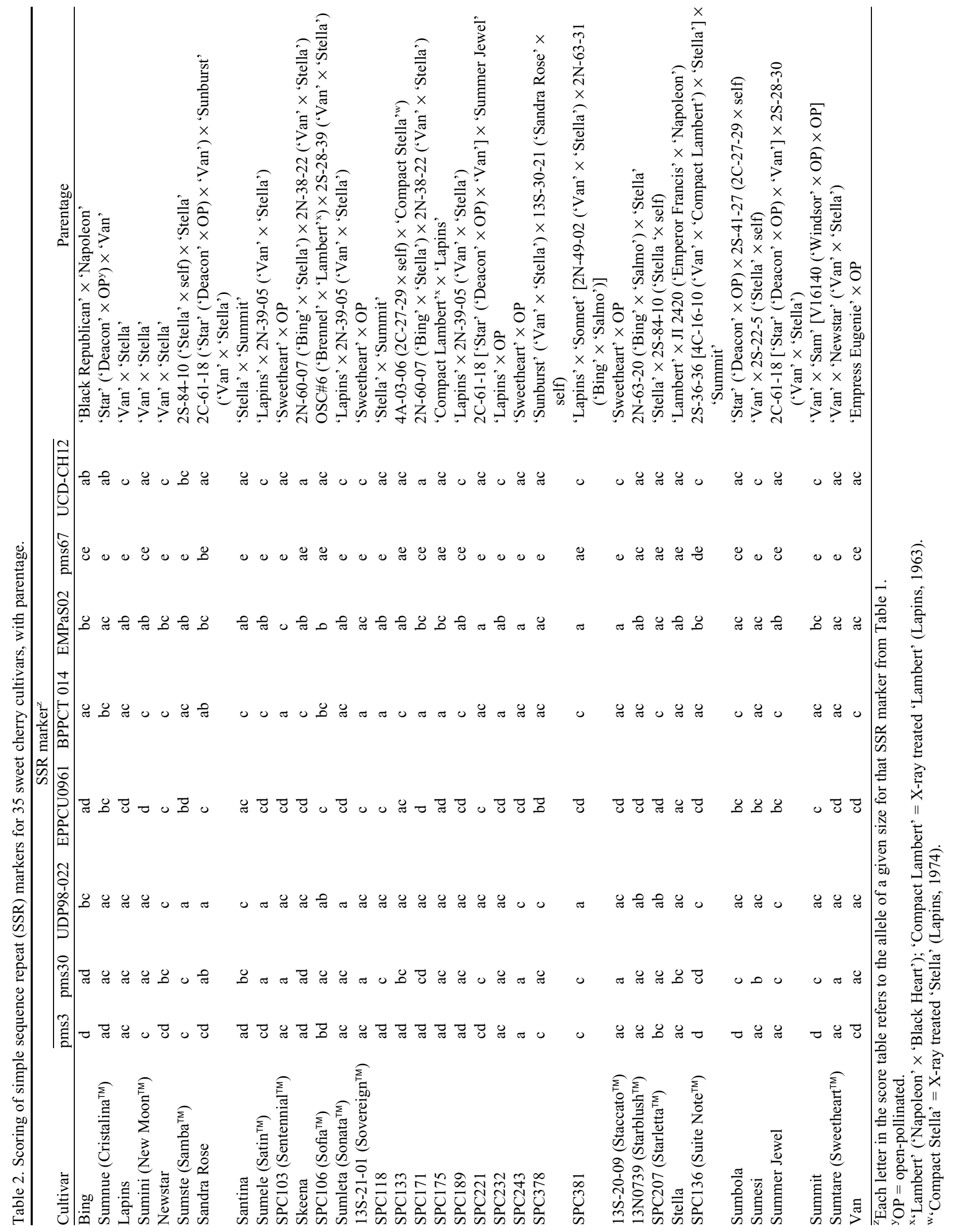




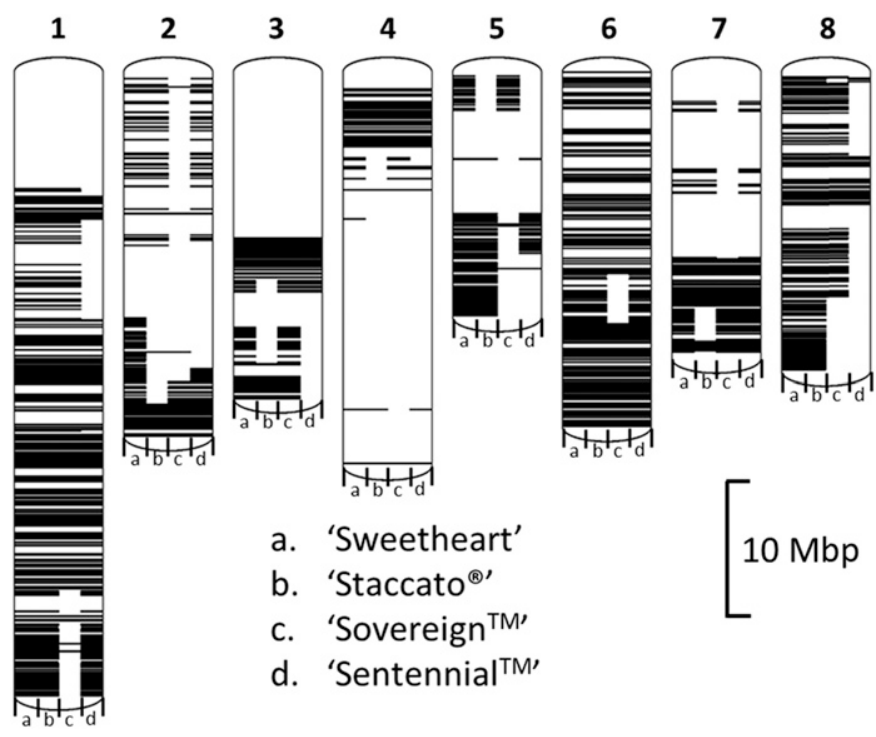

Fig. 1. Location of single nucleotide polymorphism (SNP) markers in 'Sweetheart' sweet cherry and segregation into three of its progeny. SNP markers identified in 'Sweetheart' are plotted as horizontal bars on the corresponding eight sweet cherry chromosomes. Each plotted chromosome is split vertically into four parts with a bar in each split showing the presence or absence of the SNP at that location for each of the four cultivars as indicated from left to right $[\mathrm{a}=$ 'Sweetheart' (i.e., the location of all found SNP markers); $\mathrm{b}=$ 'Staccato ${ }^{\circledR}, ; \mathrm{c}=$ 'Sovereign $^{\mathrm{TM}}$ ' $; \mathrm{d}=$ 'Sentennial ${ }^{\mathrm{TM}}$ ']

associated with these crosses. The parentage of 'Sweetheart' has been previously questioned (Cai et al., 2017) with the suggestion that 'Lapins' is more likely the parent (with 'Van') for this cultivar. The genotype for 'Lapins' in Table 2 is consistent at all loci for 'Van' $\times$ 'Lapins' being the correct parentage for 'Sweetheart'.

Given the set of eight SSR markers required to differentiate the most closely related of these selected cultivars, the next step was to investigate the most efficient way of using these for cultivar identification. The literature is filled with relationship statistics for use with molecular markers and a subset of these were chosen as a representative of those used in previous studies. Table 1 gives statistics for each of the eight loci chosen in this study. Because differentiation is the desired outcome, the direct \pm score for each allele between each pair of cultivars was chosen as the basis for evaluating the other statistics (most informative at the top to least informative at the bottom). The values for expected heterozygosity $\left(H_{\mathrm{e}}\right)$ were separated into two groups, higher in the top four and lower in the bottom four markers, without clear ranking within those groups. This corresponds to the selection criteria for the markers in that the top three were chosen for general discrimination, whereas the last five were chosen in screens with a few closely related cultivars. The exception to this grouping is EPPCU0961, which was selected specifically for the 'Sweetheart' vs. 'Staccato ${ }^{\circledR}$, discrimination but has the second highest $H_{\mathrm{e}}$ and also the largest difference between expected and observed heterozygosity $\left(H_{\mathrm{e}}\right.$ vs. $H_{\mathrm{o}}$ ).

Following the method of Tessier (Tessier et al., 1999), where a similar approach was used for grape cultivar identification, individual loci were added, and $x_{k}$ (sum of all $x_{j}$ ) was recalculated and minimized for each addition. Their calculations for $C_{j}$ and $D_{j}$ are related directly to the direct count differences [i.e., $C_{j}{ }^{*}(N$ choose 2$)=x_{j}=$ number of nondifferentiated pairs of cultivars]. Their equation for $D_{\mathrm{L}}$ is equivalent to DP. A minimum of six markers were required to discriminate all 35 cultivars (removing EMPaS02 and UCDCH-12 from the analysis). Because some markers were selected specifically for their discrimination of close relatives, the analysis was also tested in the opposite direction, i.e., removing loci one at a time to retain discrimination. Locus BPPCT 014 was the only locus that differentiates 'Lapins' and its selfed offspring ' $2 \mathrm{~N}-17-17$ '. pms67 was required to separate 'Lapins' and 'Sonata' ('Lapins' by a 'Lapins' sib) and either EPPCU0961 or UCDCH-12 was required to discriminate between 'Sweetheart' and 'Staccato ${ }^{\circledR}$ '. Therefore, three of these markers were absolutely required for discriminating the full set of 35. Surprisingly, complete discrimination could also be obtained with only five loci (UDP98-022, EPPCU0961, BPPCT 014, EMPaS02, and PMS67), removing pms3 and pms30, which had the highest predicted discrimination values for any of the loci.

The cultivars which were the most difficult to differentiate were within the 'Lapins' and 'Sweetheart' groups. Because more effort was expended to locate additional loci for identification in the 'Sweetheart' group three loci were found that can discriminate 'Sweetheart' and 'Staccato ${ }^{\circledR}$ ', but two loci are required to give even one difference for 'Lapins' vs. 'Sonata' and 'Lapins' vs. 'SPC232' in the 'Lapins' group. This level of identification was insufficient for the demanding requirements of Plant Breeder's Rights enforcement. It was also apparent that continuing to screen for useful SSR markers, although feasible, was not the most efficient means of meeting this requirement. An alternative method of genotyping was then evaluated to increase the discrimination power and to try to explain the low number of differentiating markers between 'Sweetheart' and 'Staccato ${ }^{\circledR}$ '.

RNA-Seq data were obtained from young leaf tissue for 'Sweetheart' and three of its open-pollinated progeny and used for SNP analysis. This is a method of reduced representation but has been shown to have a fairly high false SNP discovery rate unless the read coverage is sufficient to reduce the effect of sequencing errors (Ganal et al., 2009). Although calling SNP markers in RNA-Seq data are relatively well defined for species with reference genomes, it has only recently been extended to include less characterized genomes (Lopez-Maestre et al., 2016). The KisSplice program was run using 400 million 'Sweetheart' RNA-Seq reads and identified 15,390 DNA fragments containing putative SNP locations. These SNP markers were refined by requiring identical sequences in the genome with an overlap of 10 bases on either side of the SNP. This should eliminate splice junctions in the RNA-seq reads, which could present sequence differences that would not be in the genomic DNA. Next, those sequences with matches to multiple locations in the genome were removed. These correspond to gene family members or other repetitive sequences, and their mixed alignments could appear as false variants. This final set of 4232 SNP markers was evaluated for segregation in the 'Sweetheart' progeny. Of these, 3011 had genotype calls with a sufficient depth of coverage (DP $>100)$ to allow accurate assessment in the RNA-Seq data and this final set of SNPs was plotted in Fig. 1.

The three progeny of 'Sweetheart' used in this SNP analysis showed unique segregation of the 'Sweetheart' SNP markers. The fraction of SNP locations showing homozygous genotypes were $14 \%, 31 \%$, and $29 \%$ for 'Staccato ${ }^{\circledR}$ ', 'Sovereign' ${ }^{\mathrm{TM}}$ ', and 'Sentennial' ${ }^{\mathrm{TM}}$ ', respectively. This helps to explain the difficulty 
in finding SSR markers that were able to distinguish between 'Sweetheart' and 'Staccato ${ }^{\circledR}$ ' because there are only five small blocks in the genome where 'Staccato ${ }^{\circledR}$ ' differs from 'Sweetheart' (located at the bottom of chromosomes 2, 3, and 7 and the top of chromosomes 4 and 5). 'Staccato ${ }^{\circledR}$ ' has a considerably reduced number of homozygous loci even compared with its two sibs. All three of these cultivars are the end product of selection during the breeding process so their genotypes could be far removed from a random segregation of these sites. Large regions without SNP markers in 'Sweetheart' were found on the top of chromosomes 1 and 3; the middle of chromosomes 2, 5 , and 7; and the bottom of chromosome 4 . These regions were found in each of the progeny and probably indicate inheritance of haplotype blocks from the cultivar 'Van' which is both a parent and grandparent of 'Sweetheart'. The only location where all three progeny differ from their parent is at the bottom of chromosome 2. This region has been shown to contain several major quantitative trait loci for fruit traits (Cai et al., 2017), which would be of particular interest to the breeding program. Interestingly, the progeny differ from each other at that location with 'Staccato ${ }^{\circledR}$ ' and 'Sovereign' ${ }^{\mathrm{TM}}$ ' homozygous for one set of phased alleles whereas 'Sentennial' ${ }^{\mathrm{TM}}$ ' bears only the other allele in each location. Although the homozygous blocks were distinct, some intervening SNP markers remained after all refining criteria were applied. These were examined individually in alignments of all available reads and most appeared to be true polymorphisms. Although the possibility of unique noncrossover recombination events were considered, it is also possible that these interruptions in the sequence may instead be related to some uncertainty of assignment of contigs in this first draft of the sweet cherry genome.

At the current time neither of the two methods used in this study would be recommended for discovery of markers for DNA fingerprinting of highly related individuals. Although SSR markers can be used to discriminate sweet cherry cultivars as previously suggested, finding markers that differentiate closely related cultivars, such as the Lapins and 'Sweetheart, groups required extensive screening. In addition, this specific combination of markers would be expected to be useful only for these groups. The use of RNA-Seq for genotyping is more expensive than other reduced representation methods and although effective is not recommended for general use. Until sequencing becomes inexpensive enough for routine production of full genomes, some form of reduced representation sequencing would be appropriate for this analysis. Data from the RosBREED cherry SNP array (Hewitt et al., 2017; Peace et al., 2012) and preliminary analysis using restriction siteassociated DNA sequences [RADseq (Elshire et al., 2011); data not shown] provided confirming genotypes for the segregation of SNP markers into the 'Sweetheart' progeny. The RosBREED cherry SNP array was not originally considered in our analysis, as the broad set of cultivars used for development of the SNP markers was expected to provide too few markers for the closely related cultivars in this study. The data from supplemental file 'mmc' from Hewitt et al. (2017), however, gave 451 loci where 'Sweetheart' was heterozygous, which is significantly better than the 40 heterozygous SSR loci in the present study. Of these 'Sweetheart' SNP markers, 72 (16\%) were homozygous in 'Staccato ${ }^{\circledR}$ ' which is consistent with the RNA-seq SNP analysis. Because we are considering only the 'Sweetheart' SNP markers for segregation into its progeny this result would not be expected to show an ascertainment bias such as suggested by Hewitt et al.
(2017) when the results of the entire SNP array were considered. Both SNP array and RADseq analyses show unique regions of homozygosity in "Staccato ${ }^{\circledR}$ ' (data not shown); which were the same as those found for 'Staccato ${ }^{\circledR}$ ' in the RNA-Seq analysis of Fig. 1.

Finally, the problem remains of how to use this data for the routine analysis of sweet cherry cultivars. Two recurring needs have been the testing for trueness-to-type during propagation and distribution; and comparing potential new cultivars with previously licensed material for the initial investigation for the enforcement of breeders' rights. Both requirements often involve only a few samples at a time for which NextGen Sequencing and SNP array methods are not recommended because they rely on multiplexing or multiple samples, respectively, to reduce cost per sample. Instead, a small representative set of SNP markers can be chosen for which SNP-spanning PCR products can be analyzed in single samples. Techniques compatible with this approach include high-resolution melting (Wittwer et al., 2003), commercial assays (e.g., Semagn et al., 2014), and oligo ligation assays (Macdonald et al., 2005). To maximize the applicability of markers to the closely related cultivars in the 'Sweetheart' group, the SNP locations can include the four small regions showing differences between 'Sweetheart' and 'Staccato ${ }^{\circledR}$ '. A set of 16 markers chosen on opposite ends of the eight sweet cherry chromosomes, and therefore expected to be inherited independently, would increase the information content and would be sufficient to differentiate all 35 cultivars in this study, including the closely related cultivars in the 'Sweetheart' and 'Lapins' groups.

\section{Literature Cited}

Cai, L., R.E. Voorrips, E. van de Weg, C. Peace, and A. Iezzoni. 2017. Genetic structure of a QTL hotspot on chromosome 2 in sweet cherry indicates positive selection for favorable haplotypes. Mol. Breeding $37: 85$.

Camacho, C., G. Coulouris, V. Avagyan, N. Ma, J. Papadopoulos, K. Bealer, and T.L. Madden. 2009. BLAST+: Architecture and applications. BMC Bioinformatics 10:421.

Dirlewanger, E., P. Cosson, M. Tavaud, J. Aranzana, C. Poizat, A. Zanetto, P. Arus, and F. Laigret. 2002. Development of microsatellite markers in peach [Prunus persica (L.) Batsch] and their use in genetic diversity analysis in peach and sweet cherry (Prunus avium L.). Theor. Appl. Genet. 105:127-138.

Elshire, R.J., J.C. Glaubitz, Q. Sun, J.A. Poland, K. Kawamoto, E.S. Buckler, and S.E. Mitchell. 2011. A robust, simple genotyping-bysequencing (GBS) approach for high diversity species. PLoS One 6: e19379.

Fernandez i Marti, A., B. Athanson, T. Koepke, C. Font i Forcada, A. Dhingra, and N. Oraguzie. 2012. Genetic diversity and relatedness of sweet cherry (Prunus avium L.) cultivars based on single nucleotide polymorphic markers. Front. Plant Sci. $3: 116$.

Fils-Lycaon, B.R., P.A. Wiersma, K.C. Eastwell, and P. Sautiere. 1996. A cherry protein and its gene, abundantly expressed in ripening fruit, have been identified as thaumatinlike. Plant Physiol. 111:269273.

Ganal, M.W., T. Altmann, and M.S. Röder. 2009. SNP identification in crop plants. Curr. Opin. Plant Biol. 12:211-217.

George, M. and E. Regalado. 2003. Protocols for maize genotyping using SSR markers and data analysis, laboratory handbook. Asian Maize Biotechnol. Network, Intl. Maize Wheat Improvement Ctr. (CIMMYT), Manila, Phillippines.

Hewitt, S., B. Kilian, R. Hari, T. Koepke, R. Sharpe, and A. Dhingra. 2017. Evaluation of multiple approaches to identify genome-wide 
polymorphisms in closely related genotypes of sweet cherry (Prunus avium L.). Comput. Struct. Biotechnol. J. 15:290-298.

Jung, S., S.P. Ficklin, T. Lee, C-H. Cheng, A. Blenda, P. Zheng, J. Yu, A. Bombarely, I. Cho, S. Ru, K. Evans, C. Peace, A.G. Abbott, L.A Mueller, M.A. Olmstead, and D. Main. 2014. The genome database for Rosaceae (GDR): Year 10 update. Nucl. Acids Res. 42:D1237-D1244. Kloosterman, A.D., B. Budowle, and P. Daselaar. 1993. PCR-amplification and detection of the human D1S80 VNTR locus. Amplification conditions, population genetics and application in forensic analysis. Intl. J. Legal Med. 105:257-264.

Lapins, K. 1963. Note on compact mutants of Lambert cherry produced by ionizing radiation. Can. J. Plant Sci. 43:424-425.

Lapins, K.O. 1974. Compact Stella sweet cherry. Can. J. Plant Sci. 54:849-850.

Li, H. 2011. A statistical framework for SNP calling, mutation discovery, association mapping and population genetical parameter estimation from sequencing data. Bioinformatics 27:2987-2993.

Li, H. and R. Durbin. 2010. Fast and accurate long-read alignment with Burrows-Wheeler transform. Bioinformatics 26:589-595.

Lopez-Maestre, H., L. Brinza, C. Marchet, J. Kielbassa, S. Bastien, M. Boutigny, D. Monnin, A.E. Filali, C.M. Carareto, C. Vieira, F. Picard, N. Kremer, F. Vavre, M-F. Sagot, and V. Lacroix. 2016. SNP calling from RNA-seq data without a reference genome: Identification, quantification, differential analysis and impact on the protein sequence. Nucl. Acids Res. 44:e148.

Macdonald, S.J., T. Pastinen, A. Genissel, T.W. Cornforth, and A.D. Long. 2005. A low-cost open-source SNP genotyping platform for association mapping applications. Genome Biol. 6:R105.

Nei, M. 1973. Analysis of gene diversity in subdivided populations. Proc. Natl. Acad. Sci. USA 70:3321-3323.

Noli, E., M.S. Teriaca, and S. Conti. 2013. Criteria for the definition of similarity thresholds for identifying essentially derived varieties. Plant Breeding 132:525-531.

Nybom, H., K. Weising, and B. Rotter. 2014. DNA fingerprinting in botany: Past, present, future. Investig. Genet. 5:1.

Olmstead, J.W., A.M. Sebolt, A. Cabrera, S.S. Sooriyapathirana, S. Hammar, G. Iriarte, D.C. Wang, C.Y. Chen, E.d. Knaap, and A.F. Iezzoni. 2008. Construction of an intra-specific sweet cherry (Prunus avium L.) genetic linkage map and synteny analysis with the Prunus reference map. Tree Genet. Genomes 4:897-910.
Peace, C., N. Bassil, D. Main, S. Ficklin, U.R. Rosyara, T. Stegmeir, A. Sebolt, B. Gilmore, C. Lawley, T.C. Mockler, D.W. Bryant, L. Wilhelm, and A. Iezzoni. 2012. Development and evaluation of a genome-wide 6K SNP array for diploid sweet cherry and tetraploid sour cherry. PLoS One 7:e48305.

Pedersen, B.H. 2006. DNA fingerprints of 51 sweet and sour Prunus accessions using simple sequence repeats. J. Hort. Sci. Biotechnol. 81:118-124.

Pritchard, L., J.A. White, P.R.J. Birch, and I.K. Toth. 2006. GenomeDiagram: A python package for the visualization of large-scale genomic data. Bioinformatics 22:616-617.

Sacomoto, G.A., J. Kielbassa, R. Chikhi, R. Uricaru, P. Antoniou, M.F. Sagot, P. Peterlongo, and V. Lacroix. 2012. KISSPLICE: De-novo calling alternative splicing events from RNA-seq data. BMC Bioinformatics 13(suppl. 6):S5.

Semagn, K., R. Babu, S. Hearne, and M. Olsen. 2014. Single nucleotide polymorphism genotyping using kompetitive allele specific PCR (KASP): Overview of the technology and its application in crop improvement. Mol. Breeding 33:1-14.

Shirasawa, K., K. Isuzugawa, M. Ikenaga, Y. Saito, T. Yamamoto, H. Hirakawa, and S. Isobe. 2017. The genome sequence of sweet cherry (Prunus avium) for use in genomics-assisted breeding. DNA Res. 24:499-508.

Tessier, C., J. David, P. This, J.M. Boursiquot, and A. Charrier. 1999. Optimization of the choice of molecular markers for varietal identification in Vitis vinifera L. Theor. Appl. Genet. 98:171-177.

Waits, L.P., G. Luikart, and P. Taberlet. 2001. Estimating the probability of identity among genotypes in natural populations: Cautions and guidelines. Mol. Ecol. 10:249-256.

Wittwer, C.T., G.H. Reed, C.N. Gundry, J.G. Vandersteen, and R.J. Pryor. 2003. High-resolution genotyping by amplicon melting analysis using LCGreen. Clin. Chem. 49:853-860.

Wunsch, A. and J.I. Hormaza. 2004. Molecular evaluation of genetic diversity and S-allele composition of local Spanish sweet cherry (Prunus avium L.) cultivars. Genet. Resources Crop Evol. 51:635-641.

Zhou, L., F. Kappel, C. Hampson, P.A. Wiersma, and G. Bakkeren. 2002. Genetic analysis and discrimination of sweet cherry cultivars using amplified fragment length polymorphism fingerprints. J. Amer. Soc. Hort. Sci. 127:786-792. 
Supplemental Table 1. Simple sequence repeat (SSR) markers tested for amplification in sweet cherry.

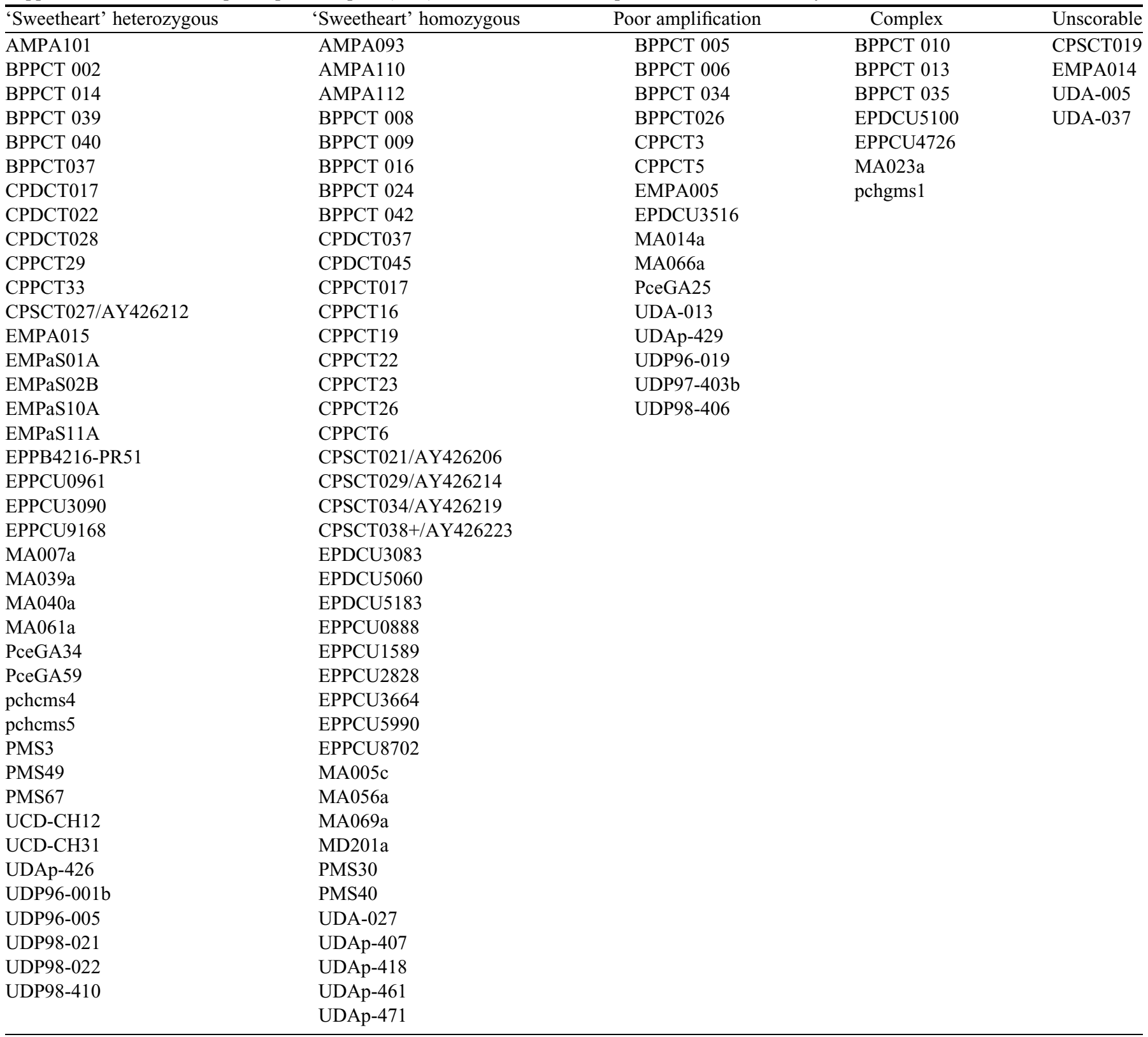

